\title{
Visual Analytics in Delirium Management
}

\author{
Werner O. HACKL ${ }^{\mathrm{a}, 1}$, Michael NETZER ${ }^{\mathrm{a}}$, Renate NANTSCHEV ${ }^{\mathrm{a}}$, Michael \\ SCHALLER $^{\mathrm{a}}$ and Elske AMMENWERTH ${ }^{\mathrm{a}}$ \\ ${ }^{a}$ UMIT - Private University for Health Sciences, Medical Informatics and Technology, \\ Institute of Medical Informatics, Hall in Tirol, Austria
}

\begin{abstract}
Background: Delirium is a patient safety issue that often occurs within the population of elderly people. As delirium may be characterized by fluctuating progress, the aim of this work is to find methods to visualize the occurrence of delirium over time in different patient stays in gerontopsychatric settings. Methods: We analyzed current data mining visualization techniques for clinical research using a delirium data set collected in a gerontopsychatric setting. Results: We identified heatmaps and dendrograms resulting from hierarchical clustering as a suitable visualization method. Conclusion: Heat maps with hierarchical clustering are a suitable data mining tool or visualization technique to study delirium cases in the time course of patient stays.
\end{abstract}

Keywords. Delirium management, patient safety, data visualization

\section{Introduction}

Gerontopsychatric wards in hospitals represent unique challenges in patient safety requirements. Delirium is one of these specific patient safety issues that often occur within the population of older people. It is associated with a massive burden to the affected people and the health care system [1,2]. Reliable risk prediction plus rapid and precise detection of cases are crucial in delirium management. Several screening instruments for detecting delirium are proposed, such as the Delirium Observation Screening Scale (DOSS) or the Confusion Assessment Method (CAM) [3]. However, their predictive ability might be limited in the psychiatric setting due to frequent side diagnoses such as dementia or depression. Therefore, in a recent study, we assessed these instruments in the gerontopsychatric setting and used different machine learning models to analyze their predictive ability. We found that CAM clearly outperformed DOSS in the specific setting and that some of our machine learning approaches were even a notch better than CAM [4]. A very important lesson learned of our study was that a horizontal view should be taken over time in addition to the pure detection of cases, as delirium can be a fluctuating condition.

Thus, this work aimed to find a suitable method to get an overview on and visualize the onset and course of delirium in the observed patient-stays.

\footnotetext{
1 Corresponding Author: Dr. Werner Hackl, UMIT TIROL, Hall in Tirol, Austria E-Mail: werner.hackl@umit-tirol.at
} 


\section{Methods}

We performed a literature analysis to identify current visualization techniques in clinical research. We found a very recent systematic overview of data mining visualization techniques including tables, charts, trees, maps, scatter plots, diagrams and graphs [5]. We evaluated these methods according to our requirements (i.e., visualization of time information). We used the statistical software $R$ [7] and the gplots package for visualization.

As dataset we used the retrospectively collected data from two gerontopsychatric wards as described in [4] (Ethical approval granted by the Ethical Committee of the Medical University of Innsbruck - EK Nr: 1032/2019). In total, 732 patient stays from 593 distinct patients were available. During these stays DOSS $(n=2.423)$, CAM $(n=$ 2.368) and ICD-10 diagnoses of dementia $(n=2.213)$ had been collected on a regular, weekly basis [4]. The data were pre-processed to match the results of the CAM/DOSS risk assessment surveys (yes/no/maybe) with the actual conditions according to the ICD10 diagnosis as gold standard in the time sequence of each stay.

\section{Results and Discussion}

Based on the overview, we identified heatmaps as a suitable tool for visualization of delirium occurrence over time. In principle, heatmaps are tables where the color of each cell represents the magnitude of a certain value. In particular, rows represent patient-stays and columns represent the time steps. The colors of each cell represent the occurrence of delirium (yes/no) according to the gold standard or according to the result of CAM (yes/maybe/no) and DOSS (yes/no or zero to 13 points when analyzed in more granularity). The rows of the heatmaps representing patients are additionally sorted using a hierarchical clustering approach. The result of the hierarchical clustering is visualized by a dendrogram, which is a binary tree where each branch represents a cluster [6].

We could clearly identify different groups and subgroups of stays: i) stays with deliruim at admission with subgroups i.1) improvement or i.2) constant condition; ii) stays were delirium occurred during the stay, with same subgroups; iii) stays without delirium cases. Next steps will be to analyze these groups and subgroups in more detail using process mining techniques.

\section{Acknowledgement}

This research was funded by the Austrian Science Fund (FWF) [PATIS: P 29076].

\section{References}

[1] S.K. Inouye, R.G.J. Westendorp, J.S. Saczynski, Delirium in elderly people. Lancet. 383(2014), 911-22.

[2] National Clinical Guideline Centre. DELIRIUM: diagnosis, prevention and management. Clinical Guideline 103 (2010). https://www.ncbi.nlm.nih.gov/books/NBK65558/pdf/Bookshelf_NBK65558.pdf

[3] W. Hasemann, R. Kressig, D. Ermini-Fünfschilling, M. Pretto, R. Spirig, Screening, Assessment und Diagnostik von Delirien. Pflege.20(4) (2007),191-204.

[4] M. Netzer, W.O. Hackl, M. Schaller, L. Alber, Evaluating Performance and Interpretability of Machine Learning Methods for Predicting Delirium in Gerontopsychiatric Patients. IOS Press - dHealth (2020)

[5] Q. Li, Overview of Data Visualization, in: Embodying Data. Springer, 2020 pp. 17-47

[6] M. Schonlau, The clustergram: A graph for visualizing hierarchical and nonhierarchical cluster analyses, The Stata Journal 2(4) (2002), 391-402

[7] R Core Team. R: A language and environment for statistical computing. R Foundation for Statistical Computing, Vienna, Austria. http://www.R-project.org/, 2021 